\title{
Effects of Tetramethylpyrazine on Functional Recovery and Neuronal Dendritic Plasticity after Experimental Stroke
}

\author{
Jun-Bin Lin, ${ }^{1}$ Chan-Juan Zheng, ${ }^{1,2}$ Xuan Zhang, ${ }^{1}$ Juan Chen, ${ }^{3}$ Wei-Jing Liao, ${ }^{1}$ and Qi Wan ${ }^{3}$ \\ ${ }^{1}$ Department of Rehabilitation Medicine, Zhongnan Hospital of Wuhan University, Wuhan 430071, China \\ ${ }^{2}$ Department of Rehabilitation Medicine, Center of Brain Department, Hubei Xinhua Hospital, Wuhan 430015, China \\ ${ }^{3}$ Department of Physiology, School of Medicine, Wuhan University, Wuhan 430071, China
}

Correspondence should be addressed to Wei-Jing Liao; weijingliao@sina.com and Qi Wan; qwan@whu.edu.cn

Received 28 September 2014; Revised 22 December 2014; Accepted 26 December 2014

Academic Editor: Joen-Rong Sheu

Copyright (C) 2015 Jun-Bin Lin et al. This is an open access article distributed under the Creative Commons Attribution License, which permits unrestricted use, distribution, and reproduction in any medium, provided the original work is properly cited.

\begin{abstract}
The 2,3,5,6-tetramethylpyrazine (TMP) has been widely used in the treatment of ischemic stroke by Chinese doctors. Here, we report the effects of TMP on functional recovery and dendritic plasticity after ischemic stroke. A classical model of middle cerebral artery occlusion (MCAO) was established in this study. The rats were assigned into 3 groups: sham group (sham operated rats treated with saline), model group (MCAO rats treated with saline) and TMP group (MCAO rats treated with $20 \mathrm{mg} / \mathrm{kg} / \mathrm{d}$ TMP). The neurological function test of animals was evaluated using the modified neurological severity score (mNSS) at $3 \mathrm{~d}, 7 \mathrm{~d}$, and $14 \mathrm{~d}$ after MCAO. Animals were euthanized for immunohistochemical labeling to measure MAP-2 levels in the peri-infarct area. Golgi-Cox staining was performed to test effect of TMP on dendritic plasticity at $14 \mathrm{~d}$ after MCAO. TMP significantly improved neurological function at $7 \mathrm{~d}$ and $14 \mathrm{~d}$ after ischemia, increased MAP-2 level at $14 \mathrm{~d}$ after ischemia, and enhanced spine density of basilar dendrites. TMP failed to affect the spine density of apical dendrites and the total dendritic length. Data analyses indicate that there was significant negative correlation between mNSS and plasticity measured at $14 \mathrm{~d}$ after MCAO. Thus, enhanced dendritic plasticity contributes to TMP-elicited functional recovery after ischemic stroke.
\end{abstract}

\section{Introduction}

Stroke is the leading cause of long-term disability in the western world, which is a severe disease characterized by its high morbidity, mortality, disability, and recurrence [1]. It has become a heavy burden to patients, families, and societies due to the excessive costs of long hospitalizations, nursing care, and rehabilitation [2]. Ischemic stroke accounts for approximately $87 \%$ of stroke [3].

2,3,5,6-Tetramethylpyrazine (TMP, Figure 1) is an active ingredient extracted from a traditional Chinese herbal medicine Ligusticum chuanxiong Hort. and has been widely used in ischemic stroke by Chinese doctors [4]. TMP exerts pharmacological effects in multiple ways with multiple targets. TMP is reported to protect ischemia reperfusion injury of heart, brain, and kidney via reducing oxidative stress, attenuating $\mathrm{Ca}^{2+}$ overload, inhibiting apoptosis, inhibiting inflammatory reaction, and so forth [5-7]. Besides the abovementioned effects, it is also demonstrated that TMP can inhibit platelet aggregation, depress blood viscosity, and ameliorate microcirculation [8], which could be another important mechanism to treat cardiovascular and cerebrovascular diseases. Recently, it has been found that TMP could protect hepatic fibrosis by modulating multiple signal pathways [911]. Furthermore, TMP had a significant therapeutic effect on diabetic nephropathy [12], which could be mediated by downregulated expression of vascular endothelial growth factor in the kidney and reduction of lipoperoxidation [13, 14]. Additionally, TMP has been reported to have beneficial effects in various types of cancer [15-17]. Specific to ischemic stroke, according to previous studies, TMP can play a protective role through the following mechanisms: antiexcitotoxicity [18], inhibiting inflammatory reaction [19], anti-apoptosis [20], antioxidant activity [21], suppression of calcium [21], 
<smiles>Cc1nc(C)c(C)nc1C</smiles>

FIgURE 1: The structure of TMP.

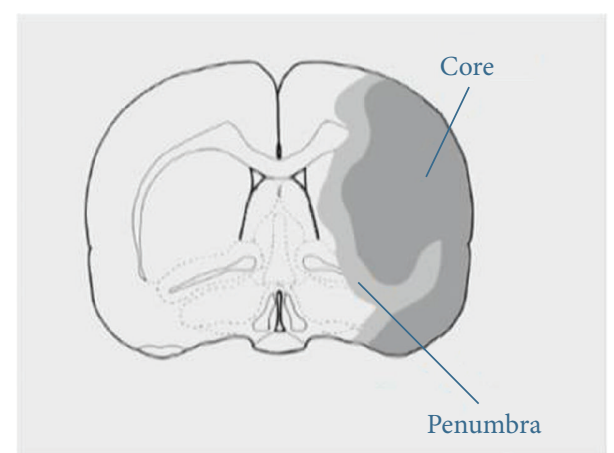

Figure 2: The schematic diagram of ischemic penumbra (IP).

thrombolytic effect [22], enhancing neurogenesis, and cell differentiation [23].

There are at least three processes during recovery after stroke: resolution of acute tissue damage, behavioral compensation, and plasticity [24]. Based on the information above, most studies focus on TMP's inhibitory roles in postischemic cascade process in acute phase. However, the effects and mechanisms of TMP on neuroplasticity are still not clear up to now. The plasticity of dendrites is an important component of plasticity $[25,26]$. When challenged by ischemic stroke, dendrites in ischemic penumbra (IP) show a series of changes with morphological modifications [27], which suggest that facilitating or optimizing the plasticity of dendrites is likely to be a promising therapeutic target. Indeed, dendritic changes after ischemic injury could be induced by drugs and rehabilitative trainings.

Ischemic penumbra (IP) was first proposed by Astrup et al. in 1981 [28]. It was defined as a region of reduced cerebral blood flow (CBF) with absent, spontaneous, or induced electrical potentials that still maintained ionic homeostasis and transmembrane electrical potentials. It has the potential for functional recovery if local blood flow can be reestablished within a limited period and is a key target for the treatment of acute stroke [29]. It is located in the peri-infarct area and Figure 2 shows schematic diagram of ischemic core and IP.

In this study, we tested the effects of TMP on functional recovery and dendritic plasticity after ischemic stroke. A classical focal cerebral ischemia reperfusion model was induced by middle cerebral artery occlusion (MCAO) in the rat and we conducted a TTC staining. Firstly, we measured the neurological function performance using the modified neurological severity score (mNSS). In order to measure the dendritic plasticity, after behavioral testing, immunohistochemistry was employed to evaluate the levels of microtubule associated protein-2 (MAP-2, marker of neuronal dendrites) and a modified Golgi-Cox staining was conducted to examine dendritic morphologic plasticity. Finally, correlations analyses between functional outcome and plasticity were performed.

\section{Materials and Methods}

2.1. Animals. A total of 78 eight-week-old male Sprague Dawley (SD) rats weighing 200-250g (purchased from Experimental Animal Center of Wuhan University, Wuhan, Hubei, China) were used for this experiment. The rats were acclimated for 3 or more days before the start of any experiments. They were housed in a controlled environment ( 4 animals per cages, $55 \pm 5 \%$ relative humidity, $22^{\circ} \mathrm{C}, 12: 12 \mathrm{~h}$ light/dark cycle) and provided with free access to food and water. All experimental procedures involving animals were approved by the Animal Care and Use Committee of Wuhan University Medical School. We made all efforts to minimize the number of animals used and their suffering.

2.2. Model. MCAO was induced using the modified intraluminal filament technique [30]. Briefly, rats were anesthetized with $10 \%$ chloral hydrate $(400 \mathrm{mg} / \mathrm{kg}$ ) intraperitoneally and, after a median incision of the neck skin, the right carotid artery (CCA), external carotid artery (ECA), and internal carotid artery (ICA) were carefully isolated. The right MCA was occluded with a monofilament nylon filament (Beijing Cinontech Biotech Co., Ltd., Beijing, China) by inserting it through the right CCA and gently advancing into the ICA up to a point approximately $17 \mathrm{~mm}$ distal to the bifurcation of the carotid artery. The filament was fixed in place and the animal was allowed to recover from anesthesia. After $2 \mathrm{~h}$, the filament was withdrawn to permit reperfusion. In sham group, all surgical procedures were the same as above without inserting a nylon filament. A heating pad was used to maintain a rectal temperature of $37.0 \pm 0.5^{\circ} \mathrm{C}$ during the surgical procedure.

$6 \mathrm{MCAO}$ rats were anesthetized with an overdose of chloral hydrate and sacrificed by decapitation at $3 \mathrm{~d}$ after MCAO. The brains were quickly removed and chilled at $-20^{\circ} \mathrm{C}$ for $10 \mathrm{~min} .2 \mathrm{~mm}$ coronal slices were cut for each brain and immersed in a PBS solution $(\mathrm{pH}=7.4)$ containing 2\% triphenyl tetrazolium chloride (TTC) (Sigma, St. Louis, $\mathrm{MO}, \mathrm{USA}$ ) at $37^{\circ} \mathrm{C}$ in the dark for $30 \mathrm{~min}$. The stained sections were then fixed in $4 \%$ paraformaldehyde for $1 \mathrm{~h}$. All stained sections were scanned and the infarct volumes were analyzed by Image Pro Plus 6.0 (Media Cybernetics Inc., Bethesda, MD, USA). To eliminate the effect of brain edema and differential shrinkage resulting from tissue processing, the percentage of infarct volume was calculated as reported previously [31].

2.3. Grouping and Administration. In this study, the animals were randomly assigned into 3 groups: sham group (sham operated rats treated with saline), model group (MCAO rats treated with saline), and TMP group (MCAO rats treated with $20 \mathrm{mg} / \mathrm{kg} / \mathrm{d}$ TMP (Aladdin Chemistry Co., Ltd., Shanghai, China)). The first administration was conducted immediately after reperfusion. All injections were conducted through 


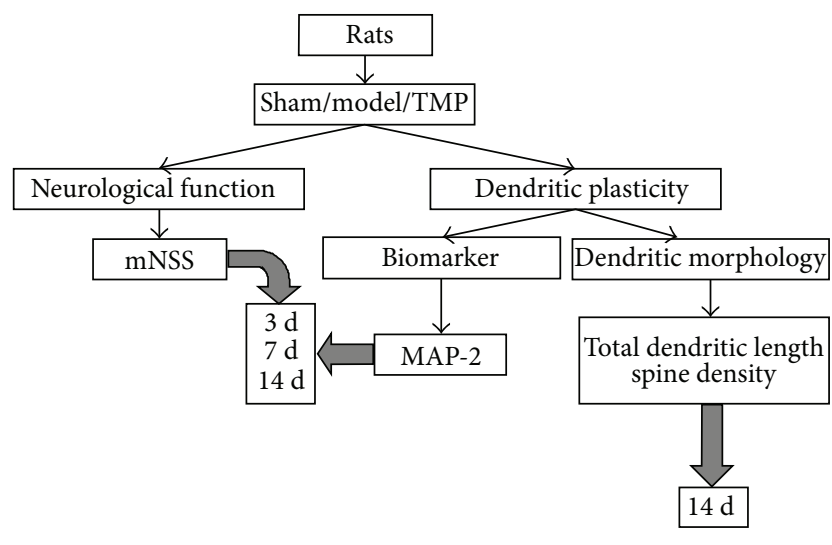

Figure 3: A simple flow-chart of experimental design.

intraperitoneal injection daily and in the volume of $5 \mathrm{~mL} / \mathrm{kg}$ until the day before they were sacrificed. After neurological function test, 54 rats were sacrificed at $3 \mathrm{~d}, 7 \mathrm{~d}$, and $14 \mathrm{~d}$ after MCAO for immunohistochemistry $(n=6$ in each group at each time point) and 18 rats for Golgi-Cox staining ( $n=6$ in each group) at $14 \mathrm{~d}$ after MCAO. A brief flow diagram is shown in Figure 3.

2.4. Neurological Function Test. Modified neurological severity score (mNSS) test [32] was measured at $3 \mathrm{~d}, 7 \mathrm{~d}$, and $14 \mathrm{~d}$ after MCAO by an observer blinded to experimental groups. The mNSS is a composite of motor, sensory, reflex, and balance tests and is graded on a scale of $0-18$ (normal score 0 , maximal deficit score 18). In the severity scores of injury, 1 score point is awarded for the inability to perform the test or for the lack of a tested reflex; thus, the higher the score is, the more severe the injury is. It is classified into three levels: 13 to 18 are graded as severe injury, 7 to 12 as moderate injury, and 1 to 6 as mild injury.

2.5. Immunohistochemistry. At $3 \mathrm{~d}, 7 \mathrm{~d}$, and $14 \mathrm{~d}$ after MCAO, rats in each group at each time point $(n=6)$ were anesthetized with an overdose of chloral hydrate and transcardially perfused with $150 \mathrm{~mL}$ of $0.9 \%$ saline followed by $150 \mathrm{~mL}$ of $4 \%$ paraformaldehyde. The brains were removed and postfixed in $4 \%$ paraformaldehyde overnight. Thereafter, paraffin embedded blocks (bregma: -2 to $+2 \mathrm{~mm}$ ) were obtained and sliced into sections of $6 \mu \mathrm{m}$ and mounted onto the polylysinecoated slides. Streptavidin-peroxidase (S-P) method [33] was adopted for immunostaining: (1) tissue sections were deparaffinized with xylene and rehydrated in ethanol; (2) they were incubated in endogenous peroxidase blocking solution (Maixin Technology Co., Ltd., Fuzhou, Fujian, China) for $10 \mathrm{~min}$ at room temperature; (3) after being incubated with normal rabbit serum (Maixin Technology Co., Ltd., Fuzhou, Fujian, China), the brain sections were incubated overnight with rabbit anti-MAP-2 antibody (1:200, Boster, Wuhan, Hubei, China) at $4^{\circ} \mathrm{C}$; (4) the sections were incubated with biotin-conjugated second antibody (Maixin Technology Co., Ltd., Fuzhou, Fujian, China) for $15 \mathrm{~min}$; (5) they were incubated with HRP-Streptavidin-Peroxidase (Maixin Technology Co., Ltd., Fuzhou, Fujian, China) for $15 \mathrm{~min}$; (6) the sections were stained with $3,3^{\prime}$-diaminobenzidine and $\mathrm{H}_{2} \mathrm{O}_{2}$, washed with tap water, and counterstained with hematoxylin. The sections were rinsed with phosphate-buffered saline (PBS, $\mathrm{pH}=7.4$ ) 3 times for 3 min between every procedure of staining. Finally, the sections were dehydrated and coverslipped. To investigate the specificity of the reactions, negative controls were established by replacing the primary antibody with PBS and normal rabbit serum.

For quantitative analysis, three randomly selected sections of each subject and five visual fields (400x) from each section in peri-infarct area were randomly captured under a microscope using a digital camera. Integrated optical density (IOD) was measured using Image Pro Plus 6.0 (Media Cybernetics Inc., Bethesda, MD, USA) for analysis. The analysis procedure was conducted by an investigator in a blind fashion.

2.6. Golgi-Cox Staining Procedure. At $14 \mathrm{~d}$ after MCAO, rats in each group $(n=6)$ were injected intraperitoneally with a lethal dose of chloral hydrate to induce anesthesia. Remove the brains as soon as possible without perfusion and rinse tissue in double distilled water for 2-3 seconds to remove blood from the surface. Hito Golgi-Cox OptimStain Kit (Hitobiotec Inc., Wilmington, DE, USA) was applied for tissue preparation and staining procedure. The whole GolgiCox staining procedure was conducted in strict accordance with the manufacturer's user manual and material safety data sheet. A series of $100 \mu \mathrm{m}$ thick coronal sections was sliced from the caudal forelimb region of the motor cortex (approximately from bregma to $+2.0 \mathrm{~mm}$ from bregma) [34] using a microtome (Leica CM1950 cryostat; Leica Biosystems $\mathrm{GmbH}$, Wetzlar, Germany).

2.7. Selection Criteria for Pyramidal Cells. To be included for analysis, neurons should be selected according to specific criteria [35]: (1) the dendritic trees had to be well impregnated to facilitate accurate observation and analysis; (2) the cell bodies and dendrites had to be in full view and not obscured by other blood vessels, astrocytes, or clustering of dendrites from other pyramidal cells; (3) they also had to appear intact and visible in the plane of section.

2.8. Sholl Analysis. To acquire images for analyzing, layer V pyramidal cells within peri-infarct area were traced at $200 \mathrm{x}$ magnification. Pyramidal neurons were readily identified by their characteristic triangular soma-shape, apical dendrites extending toward the pial surface, and numerous dendritic spines [36]. In order to measure the length of dendrites, Sholl analysis [37] was conducted using a Sholl analysis plug-in (available at http://fiji.sc/Sholl_Analysis) for Image J software (National Institutes of Health, Bethesda, MD, USA). The number of intersections of dendrites with a series of concentric rings at $20 \mu \mathrm{m}$ intervals from the centre of the cell body was counted for each cell. A reflection of total dendritic length can be determined by multiplying the number of 


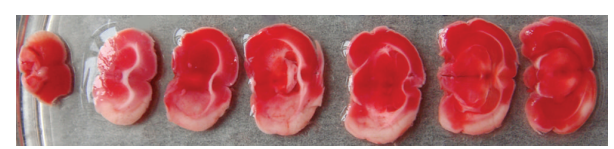

FIGURE 4: A representative photograph of TTC staining of MCAO rat.

intersections by 20 [38]. Five cells per rat were measured for statistical analysis.

2.9. Measurement of Spine Density. Dendritic spine density was analyzed from layer $\mathrm{V}$ pyramidal neurons within periinfarct area. For each cell, at least $30 \mu \mathrm{m}$ long segments of terminal basilar densities (third order or greater, $n=5$ ) and apical densities (lower half of the apical segments, $n=5$ ) on the same cell were traced at 1000x magnification [39]. The number of spines was counted and the exact length of the dendritic segment was calculated to yield spines $/ 10 \mu \mathrm{m}$ data [39]. We did not make any attempt to correct for spines hidden by the overlying dendrites. Therefore the data may be likely to underestimate the actual density.

2.10. Statistical Analysis. All data was expressed as mean \pm standard deviation (SD) and analyzed using SPSS 19.0 software (SPSS Inc., Chicago, IL, USA). Behavior data and immunohistochemical data were analyzed using repeated measures analysis of variance (rANOVA) and when the assumptions of sphericity were violated (Mauchly's test, $P<$ 0.05), the Greenhouse-Geisser correction was applied. Post hoc analyses used group designed $t$-test and Turkey's test. One-way analysis of variance (ANOVA) and Tukey's test were used for analyzing dendritic morphological data. Correlations analysis between functional outcome and plasticity were performed using the Spearman correlation coefficients. $P<$ 0.05 was considered statistically significant.

\section{Results}

3.1. TTC for Model Rats. Figure 4 shows a typical photograph of coronal sections of MCAO rat. The infarct region appeared white, and the normal tissue was red. Rats after MCAO exhibited obvious infarction which was located in cortex and striatum. The infarct volume was $38.42 \pm 4.42 \%$.

3.2. Neurological Functional Assessment. As shown in Figure 5, for model group and TMP group, rats showed functional improvement with time going on. Repeated measures analysis of variance showed significant group effects $(F=11.621, P=0.003)$. TMP treatment significantly improved functional recovery, as evidenced by improved mNSS at $7 \mathrm{~d}$ (model: $10.92 \pm 1.68$ versus TMP: $9.33 \pm 1.72$; $t=2.281, P=0.033$; decreased 14.56\%) and $14 \mathrm{~d}$ (model: $8.42 \pm 1.38$ versus TMP: $6.42 \pm 1.16 ; t=3.839, P=0.001$; decreased $23.75 \%$ ) compared with model group. However, there was no significant difference between the two groups at $3 \mathrm{~d}$ after MCAO (model: $12.75 \pm 1.66$ versus TMP:

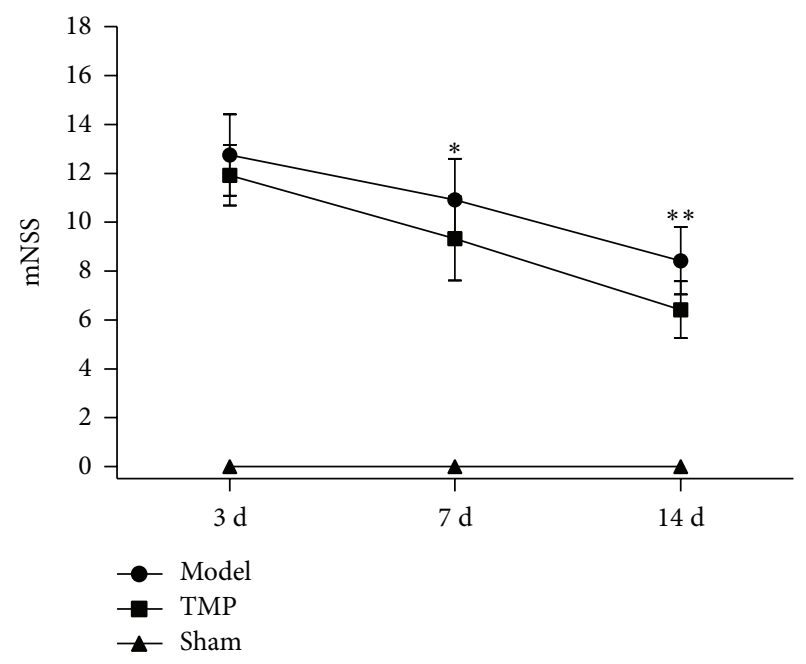

FIGURE 5: Effect of TMP on neurological status in rats with ischemic cerebral injury. The data were presented as mean \pm standard deviation $(n=12) .{ }^{*} P<0.05$ between model group and TMP group; ${ }^{* *} P<0.01$ between model group and TMP group.

$11.92 \pm 1.24 ; t=1.394, P=0.177)$. All rats in sham group performed very well without any neurological deficit.

3.3. MAP-2 Expression. In this study, IOD values were applied to indicate the expression of MAP-2 (Figure 6). In sham group, obvious MAP-2 immunostaining was observed in the dendrites of the cells. Repeated measures analysis of variance showed there was significant group effects $(F=$ 77.753, $P<0.001)$. Post hoc analyses showed that there were significant differences between three groups at $3 \mathrm{~d}$ (sham: $38635.39 \pm 2649.21$ versus model: $17958.93 \pm 1244.88$ versus TMP: $19128.20 \pm 1795.69 ; F=205.913, P<0.001), 7 \mathrm{~d}$ (sham: $38009.15 \pm 2715.61$ versus model: $22635.95 \pm 2102.93$ versus TMP: $25521.22 \pm 1764.14 ; F=80.61, P<0.001)$, and $14 \mathrm{~d}$ (sham: $39059.86 \pm 2831.29$ versus model: $31203.85 \pm$ 2478.53 versus TMP: $37147.30 \pm 2168.38 ; F=16.017, P<$ $0.001)$. Compared to sham group, rats in model group showed significantly lower expression of MAP-2 (3d, $7 \mathrm{~d}$, and $14 \mathrm{~d}$ all $P<0.001$; decreased 53.52\%, 40.45\%, and $20.11 \%$, resp.), although they exhibited an increasing trend from $3 \mathrm{~d}$ to $14 \mathrm{~d}$ after MCAO. TMP treatment resulted in upregulation in MAP-2 expression in peri-infarct area compared to model group at $14 \mathrm{~d}(P=0.003$; increased $19.05 \%)$ after MCAO.

3.4. Dendritic Morphology. The morphological analysis presented here is based on a total of 180 neurons from 18 animals. Golgi-Cox staining clearly filled the dendritic shafts (Figure 7) and the spines of neurons from layer $\mathrm{V}$ pyramidal neurons. The total dendritic length and dendritic spine density were obtained for analysis.

3.4.1. Total Dendritic Length. There was no significant difference between three groups at $14 \mathrm{~d}$ after MCAO by a one-way 

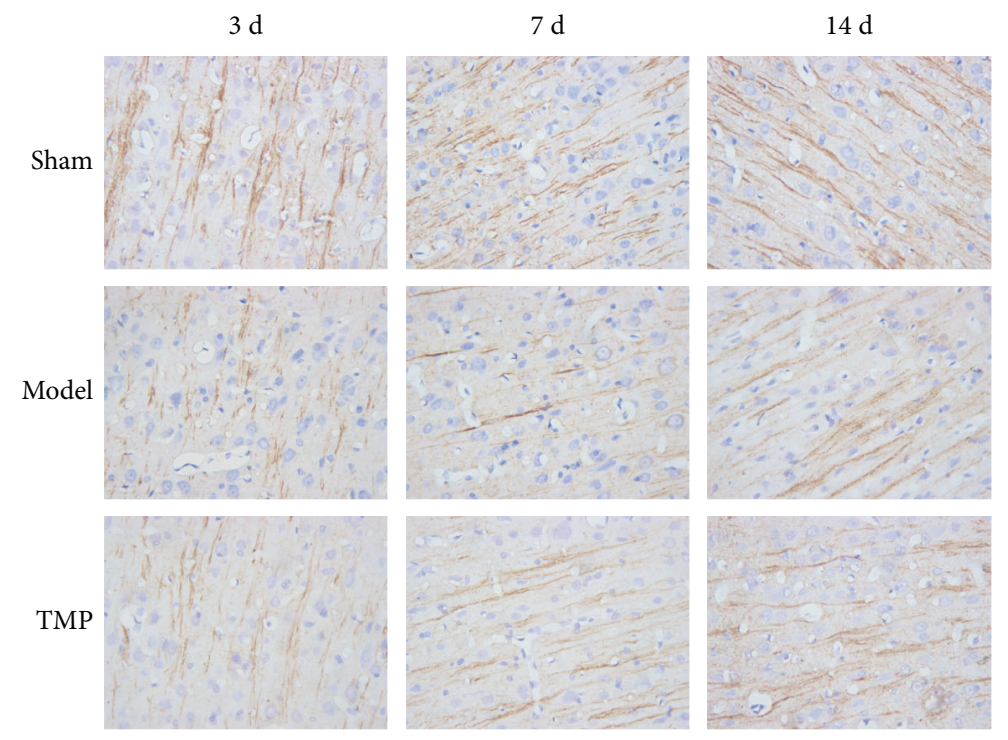

(a)

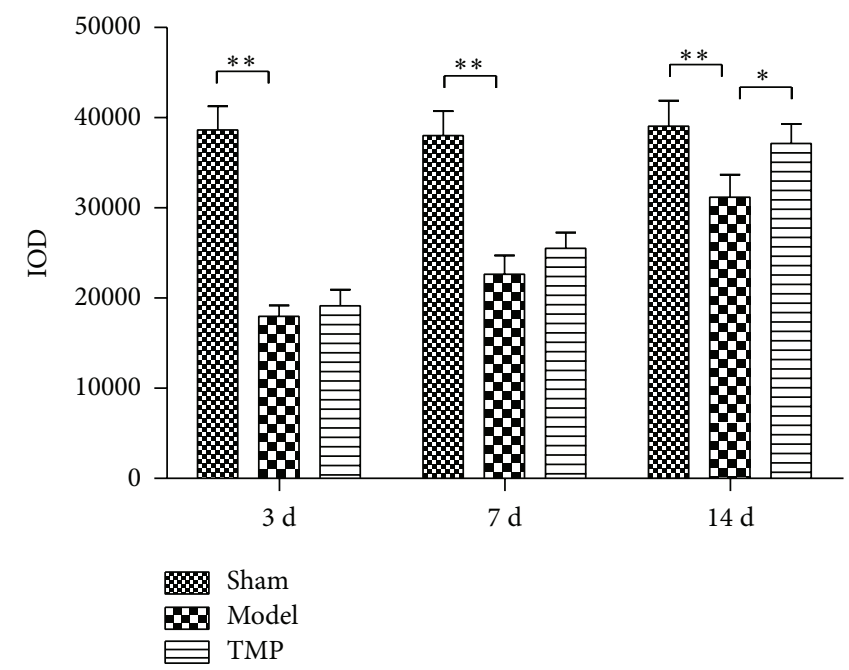

(b)

FIGURE 6: The expression levels of MAP-2 within peri-infarct area of three groups in sham, model, and TMP groups at $3 \mathrm{~d}, 7 \mathrm{~d}$, and $14 \mathrm{~d}$ after MCAO. (a) Immunohistochemical staining of three groups (400x). (b) MAP-2 levels of three groups through measuring the integral optical density (IOD). Data were presented as mean \pm standard deviation $(n=6) .{ }^{*} P<0.01$ and ${ }^{* *} P<0.001$.

ANOVA (sham: $1885.67 \pm 180.73$ versus model: $1786.00 \pm$ 166.02 versus TMP: $1814.67 \pm 145.67 ; F=0.582, P=0.571$ ) (Figure 8).

3.4.2. Spine Density of Basilar Dendrites. For layer V pyramidal neurons, a one-way ANOVA of basilar dendrites spine density found difference between groups at $14 \mathrm{~d}$ after MCAO (sham: $9.43 \pm 0.85$ versus model: $7.70 \pm 0.73$ versus TMP: $9.07 \pm$ $0.84 ; F=7.642, P=0.005$ ) (Figure 9). A following Tukey's test revealed that the dendritic spine density in model group was lower than that of sham group $(P=0.006$; decreased $18.35 \%)$ and TMP treatment increased the dendritic spine density compared to model group $(P=0.027$; increased $17.79 \%)$.
3.4.3. Spine Density of Apical Dendrites. For apical dendrites, a similar trend was observed (Figure 9). A one-way ANOVA of spine density also revealed difference between groups at $14 \mathrm{~d}$ after MCAO (sham: $9.73 \pm 1.16$ versus model: $8.30 \pm$ 0.67 versus TMP: $8.73 \pm 0.85 ; F=3.870, P=0.044)$. A following Tukey's test showed a decrease in spine density of model group compared to sham group $(P=0.040$; decreased $14.70 \%)$, while no significant increase of density was found after TMP treatment $(P=0.175)$.

3.5. Correlations Analysis. The Spearman correlation coefficients test showed that there were significant negative correlations between mNSS and plasticity measured at $14 \mathrm{~d}$ after MCAO (mNSS and MAP-2: $r=-0.619, P=0.032$; 


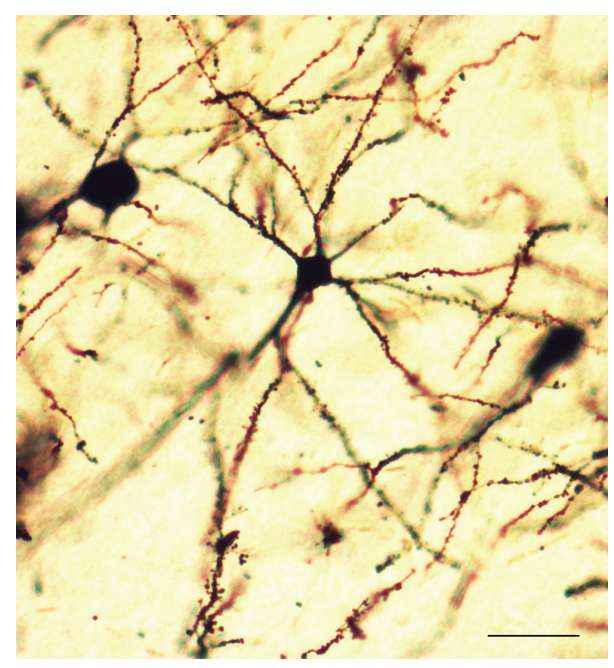

Figure 7: A representative dendritic morphology of layer V pyramidal cells of rats (Golgi-Cox staining). Photomicrograph was viewed at $\times 200$ magnification. Bar $=50 \mu \mathrm{m}$.

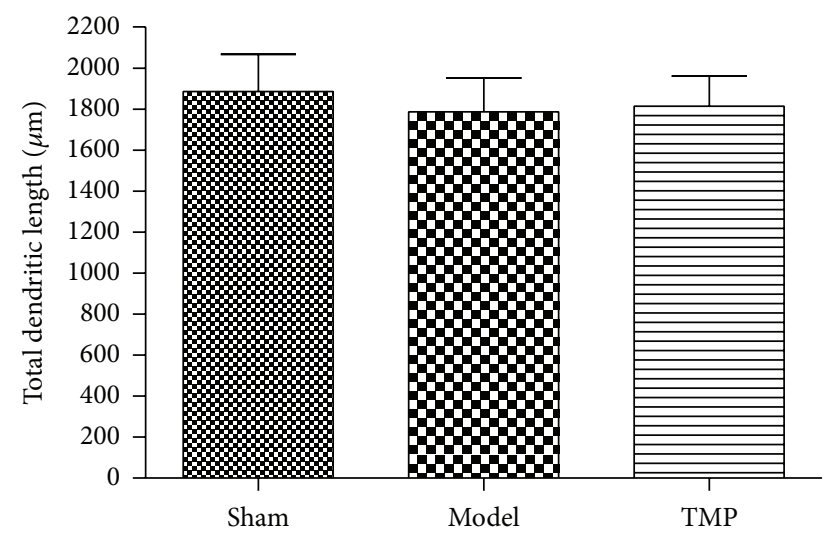

FIGURE 8: Quantification analysis of effect of TMP on total dendritic length using Sholl analysis. Data were presented as mean \pm standard deviation $(n=6)$.

mNSS and total dendritic length: $r=-0.640, P=0.025$; mNSS and spine density of basilar dendrites: $r=-0.705$, $P=0.010)$. But there was no significant correlation between mNSS and spine density of apical dendrites $(r=-0.501$, $P=0.097$ ) (Figure 10).

\section{Discussion}

MCAO model is classical model and produces obvious infarction induced by focal occlusion of middle cerebral artery [40]. TTC staining is a traditional and widely used method for the research of infarct size. In our study, relatively stable and large-sized infarction in cortex and striatum was induced by MCAO in rats in model group, which showed similar results with previous studies $[23,31]$.

Ischemic stroke often triggers a complex cascade of cellular and molecular events, including excitotoxicity, calcium overload, oxidative stress, and the following apoptosis and neuroinflammation [2]. TMP could block multiple events of the injury cascade to provide protection [19-21]. Up to now, most studies focused on the inhibitory mechanisms of TMP in the early stage of cerebral ischemia injury and only a few studies analyzed the repair mechanisms of TMP [4, 20, 23]. We reported the TMP's effects on dendritic plasticity in a relative late stage, which may provide a new target and a wider therapeutic window.

In our study, neurological score using mNSS showed obvious difference between sham and model group in all time points, which indicates that MCAO induced relative severe neurological function deficits. There must be a natural recovery process after cerebral ischemia reperfusion injury $[41,42]$, which could be confirmed by our study. TMP is a small molecular weight medicine and reported to have appreciable blood-brain barrier penetrability [43]. According to our data, TMP could improve functional outcome after focal stroke.

MAP-2 is selectively concentrated in the neuron body and dendrites, which plays a key role in maintaining neuroarchitecture, cellular differentiation, and structural and functional plasticity [30]. MAP-2 has an intimate relationship with ischemic cerebral injury and is considered to be an indication of compensatory dendrites reconstruction in remaining neurons $[44,45]$. Several studies revealed that the expression of MAP-2 decreased after ischemic cerebral injury [46-48]. In our study, in sham group, MAP-2(+) cells showed staining mainly in the dendrites of the cells; in ischemic animals, we examined the expression of MAP-2 in periinfarct area at $3 \mathrm{~d}, 7 \mathrm{~d}$, and $14 \mathrm{~d}$ after MCAO; the level of MAP-2 markedly decreased compared to sham group and persistently increased from $3 \mathrm{~d}$ to $14 \mathrm{~d}$ after stroke, which was consistent with previous study [48]. These results indicated that the expression of MAP-2 showed a dynamic process after stroke (decreasing in early stage and increasing gradually), which may represent degeneration and reconstruction of dendritic structure. Two studies $[25,49]$ declared there were a peak point and following downtrend during dendrites reconstruction. However, we did not observe this process which may be due to the relatively short period of observation.

Our data showed that treatment of TMP significantly increased MAP-2 expression level in peri-infarct area after stroke and the neurological function was improved meanwhile, indicating that promotion of the reconstruction of dendrites may contribute to the improvements of neurological function. The mechanism is not clear but may be associated with inhibition of calpains. Calpains could be activated by elevated levels of intracellular calcium after ischemic injury $[50,51]$, causing proteolysis of numerous neuronal cytoskeletal and regulatory proteins. The increase in calpain expression in the ischemic area was accompanied by a loss of its substrate MAP-2 [52]. TMP is a calcium antagonist and could markedly reverse the increased intercellular free calcium concentration [21]. This effect may contribute to upregulation of MAP-2 level. Correlation analysis showed that there was a significant negative correlation between mNSS and expression of MAP-2, indicating that TMP's effect on improvement of neurological function may be the association with upregulation of MAP-2. 


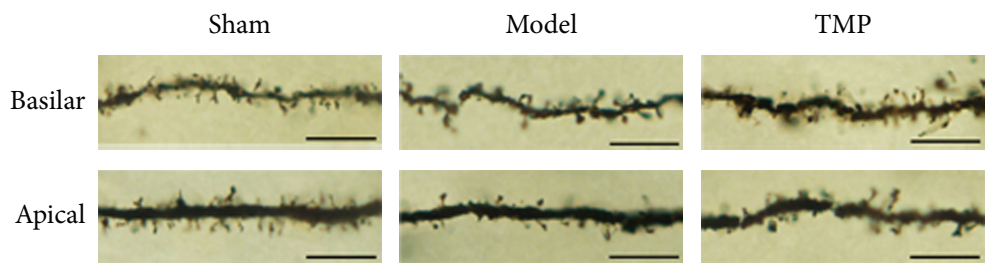

(a)

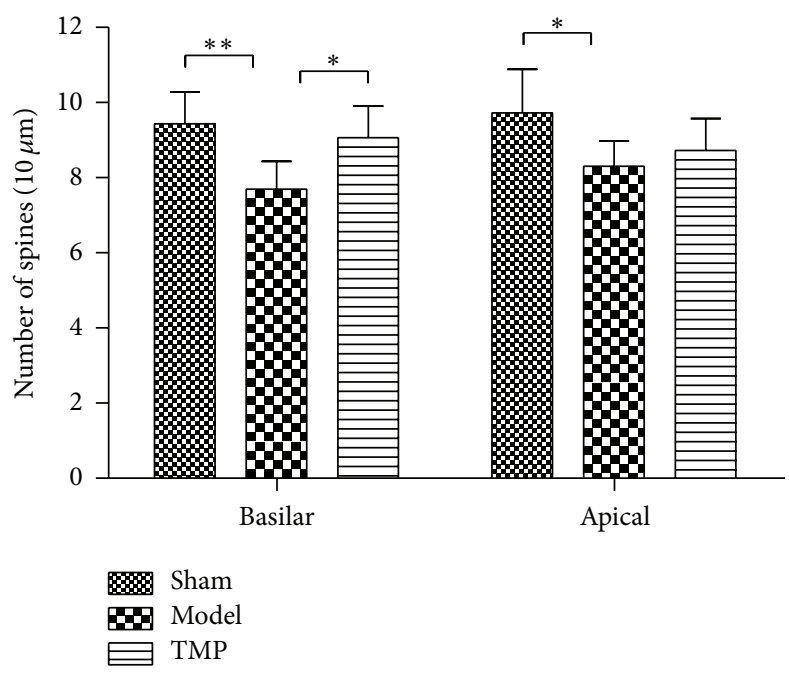

(b)

FIGURE 9: Quantification analyses of effect of TMP on dendritic spine density (basilar dendrites and apical dendrites, resp.). (a) The segments were acquired from layer V pyramidal cells and viewed at $\times 1000$ magnification. Scale bar $=10 \mu \mathrm{m}$ for all segments. (b) The dendritic spine density was expressed as spines $/ 10 \mu \mathrm{m}$ and the data were presented as mean \pm standard deviation $(n=6) .{ }^{*} P<0.05$ and ${ }^{* *} P<0.01$.

MAP-2 is an indirect marker which can be used for representing dendritic plasticity. However, morphological study is more distinct and more direct for assessments of dendrites. Golgi-Cox staining method has been used broadly for studying morphology of neurites, including quantitative analysis of dendritic length, arborization, and spine density [53], of which spine density is the most important parameter. Dendritic length reflected the total space for synapses and spine density represented the density of excitatory synapses to some extent [54]. Sholl analysis was a classical method for measuring dendritic length, which is an important parameter reflecting dendritic plasticity. We found that the dendritic length of layer $\mathrm{V}$ pyramidal cells within peri-infarct area did not change compared to sham group. In fact, the evidence about changes of dendritic length after stroke is controversial; some studies found a shortening of dendrites after cortical lesions $[38,55]$; another study found no difference or extension of dendrites in peri-infarct cortex after MCAO [56]. Such paradoxical results are perhaps associated with the absence of a peri-infarct baseline or absence of dynamic study. Brown et al. [57] conducted a longitudinal study and found there was a balance between dendrites extension and retraction after stroke, which may be a mechanism to explain our results. In addition, no obvious alternations of total dendritic length were observed after being treated by TMP, indicating that
TMP may fail to affect dendritic length totally at $14 \mathrm{~d}$ after stroke. Increasing of dendritic length is good for recovery of stroke, but the result is not good in this regard.

Dendrites and dentritic spines are the primary postsynaptic targets, which receive the majority of excitatory synapses [58]. Previous studies have shown that spine density could be enhanced by drugs [39] or rehabilitative training [59] after experimental stroke, which was likely to play a key role in mediating functional changes that occurred during and after stroke [27]. In our studies, the dentritic spine density of layer $\mathrm{V}$ pyramidal neurons decreased significantly in periinfarct area at $14 \mathrm{~d}$ after MCAO, indicating the degeneration of dendrites, which is in accordance with previous study [60]. After chronic treatment with TMP, the spine density of basilar dendrites increased compared to model group; for apical dendrites, there was no significant difference between model group and TMP group. One explanation is that the modifications of basilar dendrites and apical dendrites did not occur at the same time in the recovery period [61]. The degeneration and reorganization of dendritic spines is a complicated process and could be regulated through multiple mechanisms including receptors, scaffolding proteins, and regulators of the cytoskeleton $[62,63]$. However, the physiological mechanism responsible for TMP stimulating this increase is unclear in this experiment. Correlation analysis 


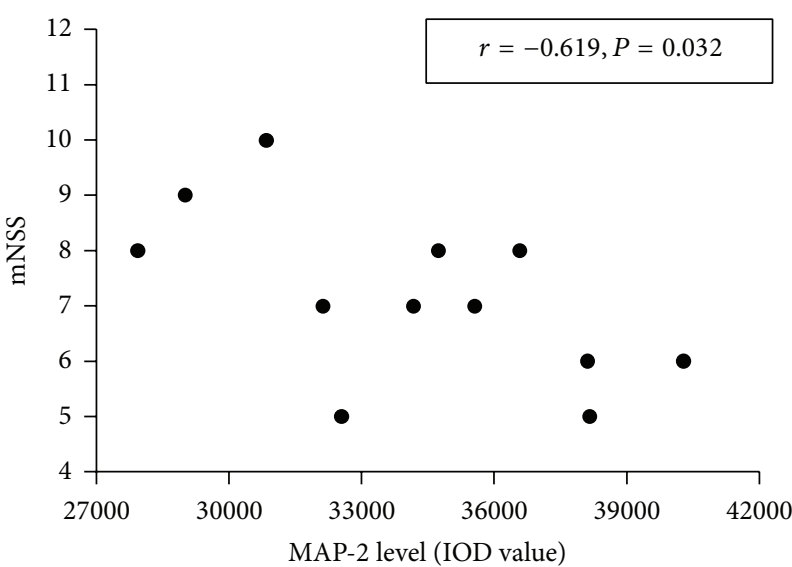

(a)

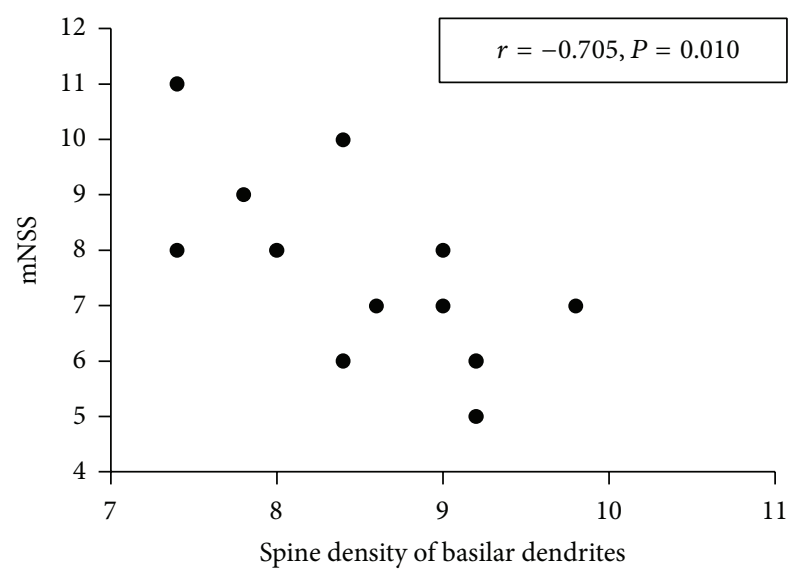

(c)

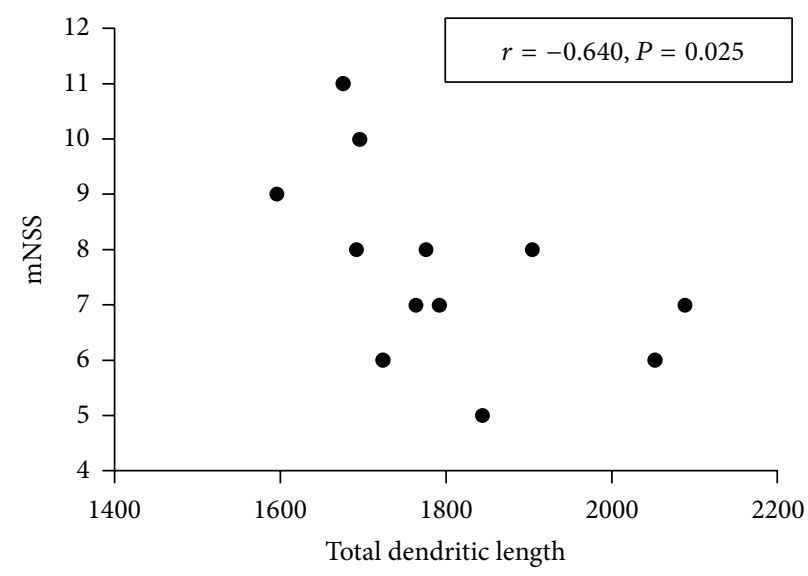

(b)

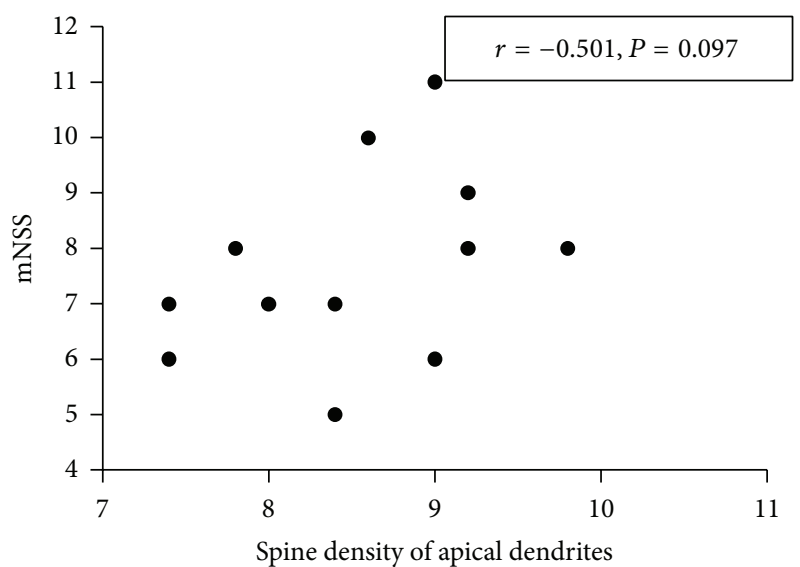

(d)

FIGURE 10: Scatterplots present correlations analysis of mNSS and plasticity measured at $14 \mathrm{~d}$ after MCAO. (a) Scatterplots of mNSS and MAP2 level. (b) Scatterplots of mNSS and total dendritic length. (c) Scatterplots of mNSS and spine density of basilar dendrites. (d) Scatterplots of mNSS and spine density of apical dendrites.

showed that there was a significant negative correlation between mNSS and spine density of basilar dendrites, indicating that TMP's effect on improvement of neurological function may be also the association with increase of spine density of basilar dendrites.

There is a dynamic change of dendrites and dendritic spine after ischemic injury over time [27]. We did not measure the dendritic morphology of other time points, so it is one of limitations that we could not reveal morphological changes during ischemic stroke and recovery.

\section{Conclusion}

TMP may increase MAP-2 level after cerebral ischemia reperfusion and decrease the alterations of neuronal dendritic spines induced by ischemia, suggesting that TMP may have a potential and specific effect on the neuronal dendritic plasticity in rats with transient focal cerebral ischemia reperfusion. Meanwhile, TMP also improved functional outcome after stroke. Taken together, after cerebral ischemia reperfusion, dendritic plasticity is one of the mechanisms that contributed to functional recovery, which might be regulated by TMP.

\section{Conflict of Interests}

The authors declare that there is no conflict of interests regarding the publication of this paper.

\section{Acknowledgment}

This study was supported by a research grant from the National Natural Science Foundation of China (no. 81072917).

\section{References}

[1] Z.-Q. Lu, Y.-J. Deng, and J.-X. Lu, "Effect of aloe polysaccharide on caspase-3 expression following cerebral ischemia and reperfusion injury in rats," Molecular Medicine Reports, vol. 6, no. 2, pp. 371-374, 2012. 
[2] E. Candelario-Jalil, "Injury and repair mechanisms in ischemic stroke: considerations for the development of novel neurotherapeutics," Current Opinion in Investigational Drugs, vol. 10, no. 7, pp. 644-654, 2009.

[3] D. Lloyd-Jones, R. J. Adams, T. M. Brown et al., "Heart disease and stroke statistics-2010 update: a report from the American Heart Association," Circulation, vol. 121, no. 7, pp. e46-e215, 2010.

[4] S.-L. Liao, T.-K. Kao, W.-Y. Chen et al., "Tetramethylpyrazine reduces ischemic brain injury in rats," Neuroscience Letters, vol. 372, no. 1-2, pp. 40-45, 2004.

[5] L. Feng, N. Ke, F. Cheng et al., "The protective mechanism of ligustrazine against renal ischemia/reperfusion injury," The Journal of Surgical Research, vol. 166, no. 2, pp. 298-305, 2011.

[6] W. Qian, X. Xiong, Z. Fang, H. Lu, and Z. Wang, "Protective effect of tetramethylpyrazine on myocardial ischemiareperfusion injury," Evidence-Based Complementary and Alternative Medicine, vol. 2014, Article ID 107501, 9 pages, 2014.

[7] Y. Chang, G. Hsiao, S. H. Chen et al., "Tetramethylpyrazine suppresses HIF-lalpha, TNF-alpha, and activated caspase-3 expression in middle cerebral artery occlusion-induced brain ischemia in rats," Acta Pharmacologica Sinica, vol. 28, no. 3, pp. 327-333, 2007.

[8] X. Cai, Z. Chen, X. Pan et al., "Inhibition of angiogenesis, fibrosis and thrombosis by tetramethylpyrazine: mechanisms contributing to the SDF-1/CXCR4 axis," PLoS ONE, vol. 9, no. 2, Article ID e88176, 2014.

[9] X. Zhang, F. Zhang, D. Kong et al., "Tetramethylpyrazine inhibits angiotensin II-induced activation of hepatic stellate cells associated with interference of platelet-derived growth factor $\beta$ receptor pathways," FEBS Journal, vol. 281, no. 12, pp. 2754-2768, 2014.

[10] F. Zhang, Z. Zhang, D. Kong et al., "Tetramethylpyrazine reduces glucose and insulin-induced activation of hepatic stellate cells by inhibiting insulin receptor-mediated PI3K/AKT and ERK pathways," Molecular and Cellular Endocrinology, vol. 382, no. 1, pp. 197-204, 2014.

[11] F. Zhang, C. Ni, D. Kong et al., "Ligustrazine attenuates oxidative stress-induced activation of hepatic stellate cells by interrupting platelet-derived growth factor- $\beta$ receptor-mediated ERK and p38 pathways," Toxicology and Applied Pharmacology, vol. 265, no. 1, pp. 51-60, 2012.

[12] B. Wang, Q. Ni, X. Wang, and L. Lin, "Meta-analysis of the clinical effect of ligustrazine on diabetic nephropathy," The American Journal of Chinese Medicine, vol. 40, no. 1, pp. 25-37, 2012.

[13] Q.-H. Yang, Y. Liang, Q. Xu, Y. Zhang, L. Xiao, and L.-Y. Si, "Protective effect of tetramethylpyrazine isolated from Ligusticum chuanxiong on nephropathy in rats with streptozotocininduced diabetes," Phytomedicine, vol. 18, no. 13, pp. 1148-1152, 2011.

[14] L.-M. Lee, C.-F. Liu, and P.-P. Yang, "Effect of tetramethylpyrazine on lipid peroxidation in streptozotocin-induced diabetic mice," The American Journal of Chinese Medicine, vol. 30, no. 4, pp. 601-608, 2002.

[15] K. Yu, Z. Chen, X. Pan et al., "Tetramethylpyrazine-mediated suppression of C6 gliomas involves inhibition of chemokine receptor CXCR4 expression," Oncology Reports, vol. 28, no. 3, pp. 955-960, 2012.

[16] Y. Zhang, X. Liu, T. Zuo, Y. Liu, and J. H. Zhang, "Tetramethylpyrazine reverses multidrug resistance in breast cancer cells through regulating the expression and function of Pglycoprotein," Medical Oncology, vol. 29, no. 2, pp. 534-538, 2012.

[17] X.-B. Wang, S.-S. Wang, Q.-F. Zhang et al., "Inhibition of tetramethylpyrazine on P-gp, MRP2, MRP3 and MRP5 in multidrug resistant human hepatocellular carcinoma cells," Oncology Reports, vol. 23, no. 1, pp. 211-215, 2010.

[18] Y.-H. Shih, S.-L. Wu, W.-F. Chiou, H.-H. Ku, T.-L. Ko, and Y.-S. Fu, "Protective effects of tetramethylpyrazine on kainate induced excitotoxicity in hippocampal culture," NeuroReport, vol. 13, no. 4, pp. 515-519, 2002.

[19] T.-K. Kao, C.-Y. Chang, Y.-C. Ou et al., "Tetramethylpyrazine reduces cellular inflammatory response following permanent focal cerebral ischemia in rats," Experimental Neurology, vol. 247, pp. 188-201, 2013.

[20] T.-K. Kao, Y.-C. Ou, J.-S. Kuo et al., "Neuroprotection by tetramethylpyrazine against ischemic brain injury in rats," Neurochemistry International, vol. 48, no. 3, pp. 166-176, 2006.

[21] Q. Tang, R. Han, H. Xiao, J. Shen, Q. Luo, and J. Li, "Neuroprotective effects of tanshinone IIA and/or tetramethylpyrazine in cerebral ischemic injury in vivo and in vitro," Brain Research, vol. 1488, pp. 81-91, 2012.

[22] Y. Sun, J. Jiang, Z. Zhang et al., "Antioxidative and thrombolytic TMP nitrone for treatment of ischemic stroke," Bioorganic \& Medicinal Chemistry, vol. 16, no. 19, pp. 8868-8874, 2008.

[23] X. Xiao, Y. Liu, C. Qi et al., "Neuroprotection and enhanced neurogenesis by tetramethylpyrazine in adult rat brain after focal ischemia," Neurological Research, vol. 32, no. 5, pp. 547-555, 2010.

[24] S. T. Carmichael, "Plasticity of cortical projections after stroke," The Neuroscientist, vol. 9, no. 1, pp. 64-75, 2003.

[25] R. J. Nudo, "Plasticity," NeuroRx, vol. 3, no. 4, pp. 420-427, 2006.

[26] B. B. Johansson and P. V. Belichenko, "Neuronal plasticity and dendritic spines: effect of environmental enrichment on intact and postischemic rat brain," Journal of Cerebral Blood Flow \& Metabolism, vol. 22, no. 1, pp. 89-96, 2002.

[27] C. E. Brown and T. H. Murphy, "Livin' on the edge: imaging dendritic spine turnover in the peri-infarct zone during ischemic stroke and recovery," The Neuroscientist, vol. 14, no. 2, pp. 139146, 2008.

[28] J. Astrup, B. K. Siesjö, and L. Symon, "Thresholds in cerebral ischemia-the ischemic penumbra," Stroke, vol. 12, no. 6, pp. 723-725, 1981.

[29] W.-D. Heiss, "The ischemic penumbra: how does tissue injury evolve?" Annals of the New York Academy of Sciences, vol. 1268, no. 1, pp. 26-34, 2012.

[30] Q. Zhou, Q. Zhang, X. Zhao et al., "Cortical electrical stimulation alone enhances functional recovery and dendritic structures after focal cerebral ischemia in rats," Brain Research, vol. 1311, pp. 148-157, 2010.

[31] Y. M. Zhang, H. Xu, H. Sun, S. H. Chen, and F. M. Wang, "Electroacupuncture treatment improves neurological function associated with regulation of tight junction proteins in rats with cerebral ischemia reperfusion injury,", Evidence-Based Complementary and Alternative Medicine, vol. 2014, Article ID 989340, 10 pages, 2014.

[32] J. Chen, Y. Li, L. Wang et al., "Therapeutic benefit of intravenous administration of bone marrow stromal cells after cerebral ischemia in rats," Stroke, vol. 32, no. 4, pp. 1005-1011, 2001.

[33] X. Bao, X. Tian, X. Hu, Z. Zhao, Y. Qu, and C. Song, "Discovery of specific tryptophan hydroxylase in the brain of the beetle 
Harmonia axyridis," Brain Research, vol. 1073-1074, no. 1, pp. 202-208, 2006.

[34] G. Paxinos and C. Watson, The Rat Brain in Stereotaxic Coordinates, Elsevier, London, UK, 2007.

[35] C. L. R. Gonzalez, O. A. Gharbawie, P. T. Williams, J. A. Kleim, B. Kolb, and I. Q. Whishaw, "Evidence for bilateral control of skilled movements: ipsilateral skilled forelimb reaching deficits and functional recovery in rats follow motor cortex and lateral frontal cortex lesions," European Journal of Neuroscience, vol. 20, no. 12, pp. 3442-3452, 2004.

[36] F. Alcantara-Gonzalez, I. Juarez, O. Solis et al., "Enhanced dendritic spine number of neurons of the prefrontal cortex, hippocampus, and nucleus accumbens in old rats after chronic donepezil administration," Synapse, vol. 64, no. 10, pp. 786-793, 2010.

[37] D. A. Sholl, "Dendritic organization in the neurons of the visual and motor cortices of the cat," Journal of anatomy, vol. 87, no. 4 , pp. 378-406, 1953.

[38] R. L. Gibb, C. L. R. Gonzalez, W. Wegenast, and B. E. Kolb, "Tactile stimulation promotes motor recovery following cortical injury in adult rats," Behavioural Brain Research, vol. 214, no. 1, pp. 102-107, 2010.

[39] O. Hurtado, A. Cárdenas, J. M. Pradillo et al., "A chronic treatment with CDP-choline improves functional recovery and increases neuronal plasticity after experimental stroke," Neurobiology of Disease, vol. 26, no. 1, pp. 105-111, 2007.

[40] F. Liu and L. D. McCullough, "Middle cerebral artery occlusion model in rodents: methods and potential pitfalls," Journal of Biomedicine \& Biotechnology, vol. 2011, Article ID 464701, 9 pages, 2011.

[41] D. C. Morris, M. Chopp, L. Zhang, M. Lu, and Z. G. Zhang, "Thymosin $\beta 4$ improves functional neurological outcome in a rat model of embolic stroke," Neuroscience, vol. 169, no. 2, pp. 674-682, 2010.

[42] M. Song, Y.-J. Kim, Y.-H. Kim, J. Roh, S. U. Kim, and B.-W. Yoon, "Effects of duplicate administration of human neural stem cell after focal cerebral ischemia in the rat," International Journal of Neuroscience, vol. 121, no. 8, pp. 457-461, 2011.

[43] T.-H. Tsai and C.-C. Liang, "Pharmacokinetics of tetramethylpyrazine in rat blood and brain using microdialysis," International Journal of Pharmaceutics, vol. 216, no. 1-2, pp. 6166, 2001.

[44] Y. Li, N. Jiang, C. Powers, and M. Chopp, "Neuronal damage and plasticity identified by microtubule-associated protein 2 , growth-associated protein 43 , and cyclin D1 immunoreactivity after focal cerebral ischemia in rats," Stroke, vol. 29, no. 9, pp. 1972-1980, 1998.

[45] P. C. Garcia, C. C. Real, A. F. B. Ferreira, S. R. Alouche, L. R. G. Britto, and R. S. Pires, "Different protocols of physical exercise produce different effects on synaptic and structural proteins in motor areas of the rat brain," Brain Research, vol. 1456, pp. 3648, 2012.

[46] M. Sun, Y. Zhao, Y. Gu, and C. Xu, "Neuroprotective actions of aminoguanidine involve reduced the activation of calpain and caspase- 3 in a rat model of stroke," Neurochemistry International, vol. 56, no. 4, pp. 634-641, 2010.

[47] M. Sun, Y. Zhao, Y. Gu, and C. Xu, "Inhibition of nNOS reduces ischemic cell death through down-regulating calpain and caspase- 3 after experimental stroke," Neurochemistry International, vol. 54, no. 5-6, pp. 339-346, 2009.

[48] F. Wang, Z. Liang, Q. Hou et al., "Nogo-A is involved in secondary axonal degeneration of thalamus in hypertensive rats with focal cortical infarction," Neuroscience Letters, vol. 417, no. 3, pp. 255-260, 2007.

[49] T. A. Jones, S. D. Bury, D. L. Adkins-Muir, L. M. Luke, R. P. Allred, and J. T. Sakata, "Importance of behavioral manipulations and measures in rat models of brain damage and brain repair," ILAR Journal, vol. 44, no. 2, pp. 144-152, 2003.

[50] B. C. White, J. M. Sullivan, D. J. DeGracia et al., "Brain ischemia and reperfusion: molecular mechanisms of neuronal injury," Journal of the Neurological Sciences, vol. 179, no. 1-2, pp. 1-33, 2000.

[51] R. T. Bartus, R. L. Dean, K. Cavanaugh, D. Eveleth, D. L. Carriero, and G. Lynch, "Time-related neuronal changes following middle cerebral artery occlusion: implications for therapeutic intervention and the role of calpain," Journal of Cerebral Blood Flow \& Metabolism, vol. 15, no. 6, pp. 969-979, 1995.

[52] M. Liebetrau, H. Martens, N. Thomassen et al., "Calpain inhibitor A-558693 in experimental focal cerebral ischemia in rats," Neurological Research, vol. 27, no. 5, pp. 466-470, 2005.

[53] R. Gibb and B. Kolb, "A method for vibratome sectioning of Golgi-Cox stained whole rat brain," Journal of Neuroscience Methods, vol. 79, no. 1, pp. 1-4, 1998.

[54] B. Kolb, R. Brown, A. Witt-Lajeunesse, and R. Gibb, "Neural compensations after lesion of the cerebral cortex," Neural Plasticity, vol. 8, no. 1-2, pp. 1-16, 2001.

[55] R. Mostany and C. Portera-Cailliau, "Absence of large-scale dendritic plasticity of layer 5 pyramidal neurons in peri-infarct cortex," The Journal of Neuroscience, vol. 31, no. 5, pp. 1734-1738, 2011.

[56] C. L. R. Gonzalez and B. Kolb, "A comparison of different models of stroke on behaviour and brain morphology," The European Journal of Neuroscience, vol. 18, no. 7, pp. 1950-1962, 2003.

[57] C. E. Brown, J. D. Boyd, and T. H. Murphy, "Longitudinal in vivo imaging reveals balanced and branch-specific remodeling of mature cortical pyramidal dendritic arbors after stroke," Journal of Cerebral Blood Flow \& Metabolism, vol. 30, no. 4, pp. 783-791, 2010.

[58] X. Yu and Y. Zuo, "Spine plasticity in the motor cortex," Current Opinion in Neurobiology, vol. 21, no. 1, pp. 169-174, 2011.

[59] J. Biernaskie and D. Corbett, "Enriched rehabilitative training promotes improved forelimb motor function and enhanced dendritic growth after focal ischemic injury," The Journal of Neuroscience, vol. 21, no. 14, pp. 5272-5280, 2001.

[60] T. Jiang, R. X. Xu, A. W. Zhang et al., "Effects of transcranial direct current stimulation on hemichannel pannexin-1 and neural plasticity in rat model of cerebral infarction," Neuroscience, vol. 226, pp. 421-426, 2012.

[61] T. A. Jones and T. Schallert, "Overgrowth and pruning of dendrites in adult rats recovering from neocortical damage," Brain Research, vol. 581, no. 1, pp. 156-160, 1992.

[62] J. Lippman and A. Dunaevsky, "Dendritic spine morphogenesis and plasticity," Journal of Neurobiology, vol. 64, no. 1, pp. 47-57, 2005.

[63] T. Tada and M. Sheng, "Molecular mechanisms of dendritic spine morphogenesis," Current Opinion in Neurobiology, vol. 16, no. 1, pp. 95-101, 2006. 


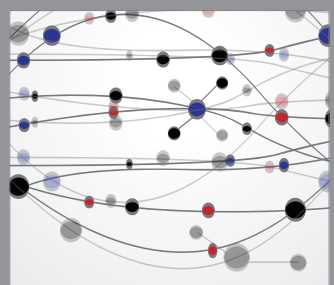

The Scientific World Journal
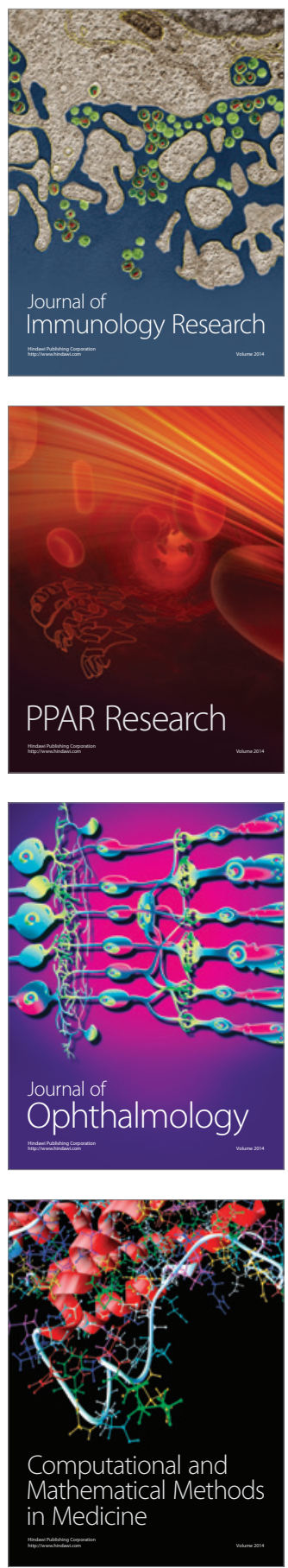

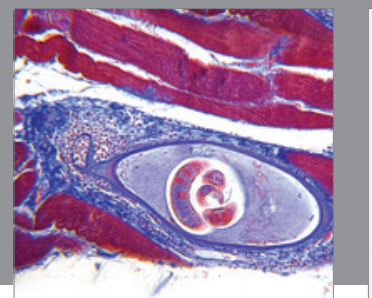

Gastroenterology

Research and Practice
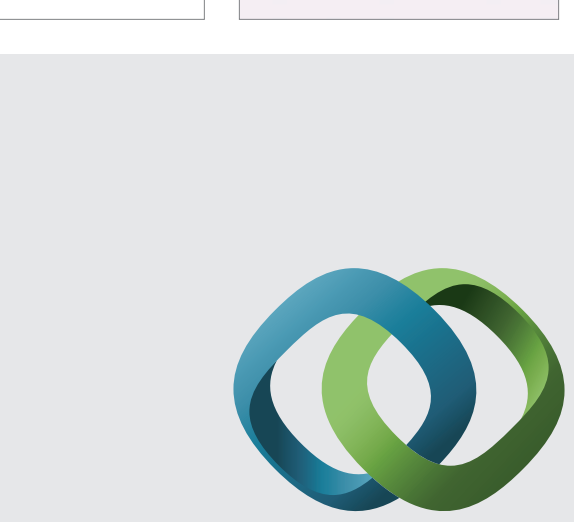

\section{Hindawi}

Submit your manuscripts at

http://www.hindawi.com
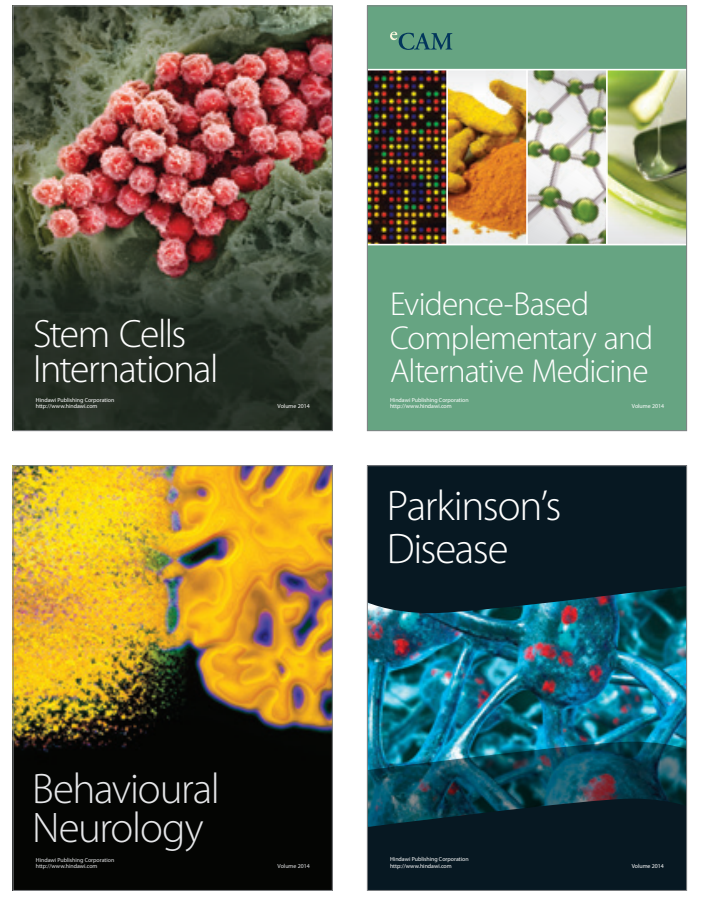
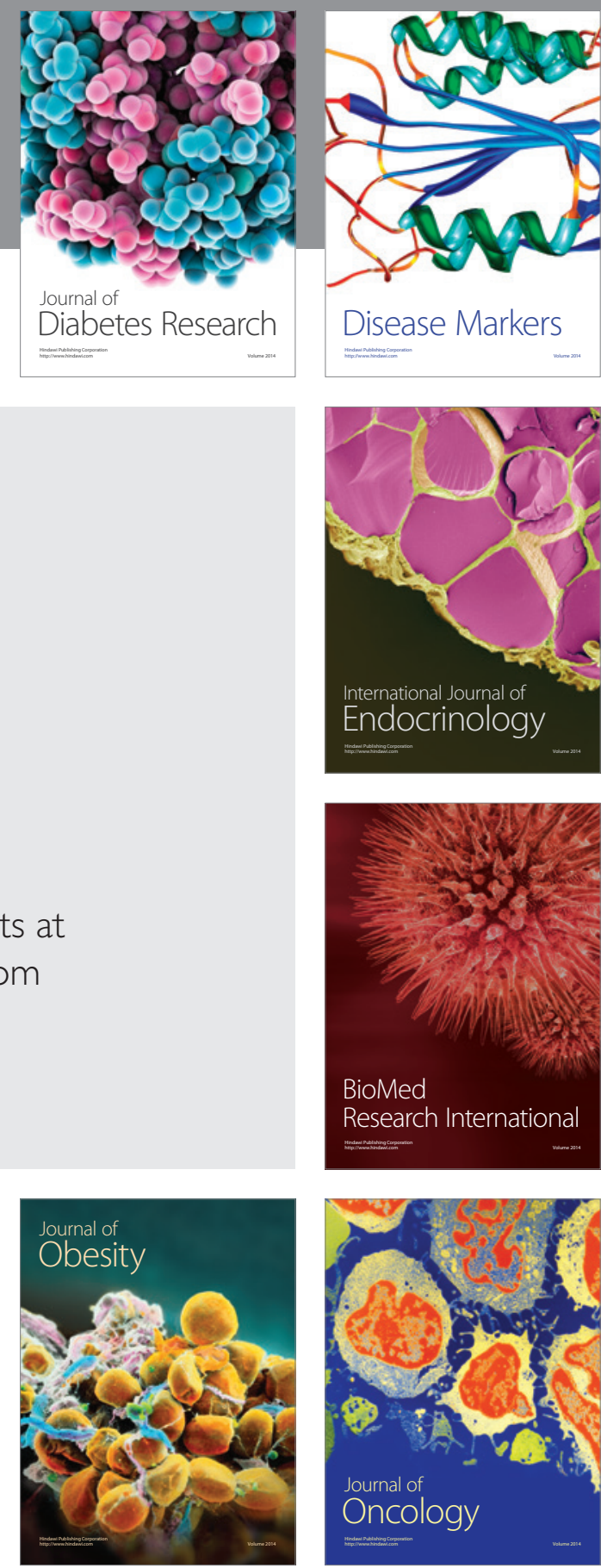

Disease Markers
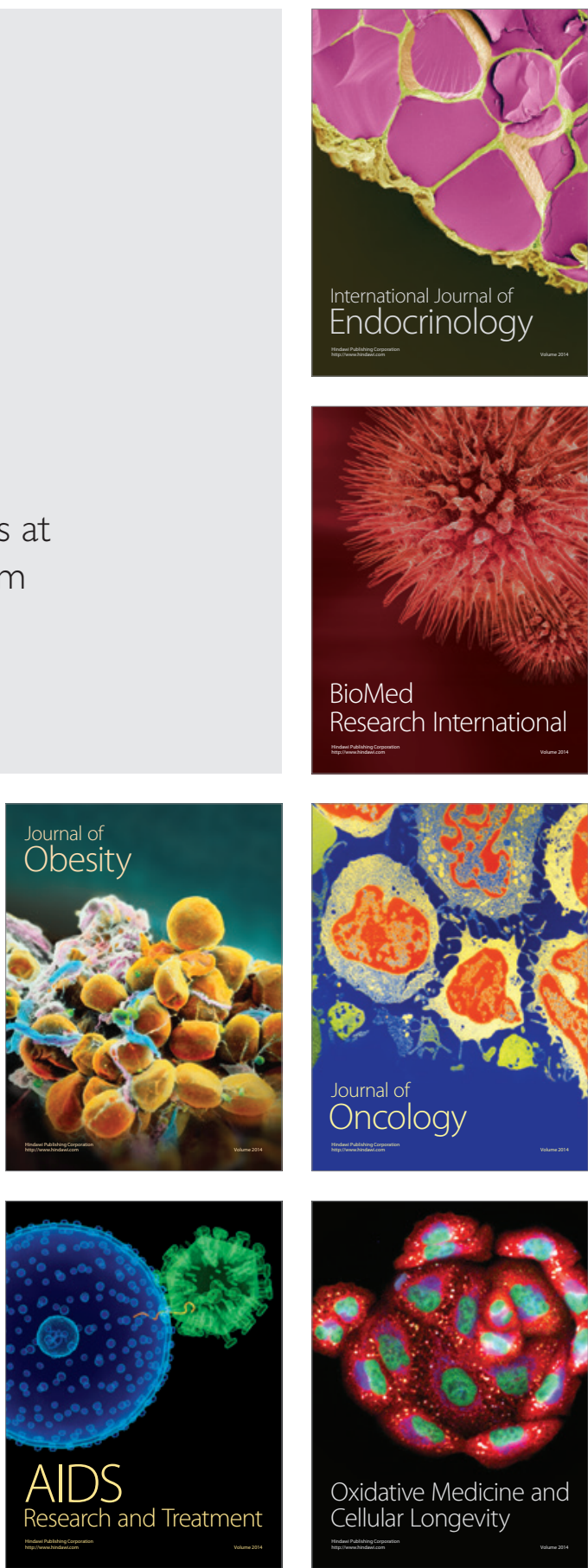